To appear in Proceedings of the International Symposium on ComputerArchitecture (ISCA), May 2002.

\title{
Transient-Fault Recovery Using Simultaneous Multithreading
}

\author{
T. N. Vijaykumar, Irith Pomeranz, and Karl Cheng
}

\begin{abstract}
We propose Simultaneously and Redundantly Threaded processors with Recovery (SRTR) that enhances a previously proposed scheme for transient error detection, called Simultaneously and Redundantly Threaded (SRT) processors, to include transient fault recovery. SRT replicates an application into two communicating threads, one executing ahead of the other. The leading thread communicates the values it produces to the trailing thread, which repeats the computation and compares the values produced by the two threads. SRT's leading instructions may commit before checking for errors, relying on the trailing thread to detect errors. SRTR, on the other hand, must not allow any leading instruction to commit before checking, since a faulty instruction cannot be undone once the instruction commits. To avoid leading instructions stalling at commit while waiting for their trailing counterparts, SRTR exploits the time between completion and commit of a leading instruction. SRTR compares the leading and trailing values as soon as the trailing instruction completes, typically before the leading instruction reaches the commit point. To avoid increasing the bandwidth demand on the register file for checking register values, SRTR uses the register value queue $(R V Q)$ to hold register values for checking. To reduce the bandwidth pressure on the RVQ itself, SRTR employs dependence-based checking elision (DBCE). By reasoning that faults propagate through dependent instructions, DBCE exploits register (true) dependence chains so that only the last instruction in a chain uses the RVQ to check leading and trailing values. The performance of SRTR is within 1\% and 7\% of the SRT performance for SPEC95 integer and floating-point programs, respectively. While SRTR without DBCE incurs about $18 \%$ performance loss when the number of RVQ ports is reduced from four (which is equivalent to an unlimited number) to two ports, with DBCE, a two-ported RVQ performs within $2 \%$ of a four-ported RVQ.
\end{abstract}

\section{Introduction}

The downscaling of feature sizes in CMOS technologies is resulting in faster transistors and lower supply voltages. While this trend contributes to improving the overall performance and reducing per-transistor power, it also implies that microprocessors are increasingly more susceptible to transient errors of various types. For instance, cosmic alpha particles may charge or discharge internal nodes of logic or SRAM cells; and lower supply voltages result in reduced noise margins allowing high-frequency crosstalk to flip bit values. The result is degraded reliability even in commodity microprocessors for which reliability has not been a concern until recently.

To address reliability issues, Simultaneously and Redundantly Threaded (SRT) processors are proposed in [8] based on the Simultaneous Multithreaded architecture (SMT) [14]. SRT detects transient errors by replicating an application into two communicating threads, one (called the leading thread) executing ahead of the other (called the trailing thread). Conceptually, the leading thread communicates the results of its computations to the trailing thread, which repeats the computations and compares the values produced by the two threads.

Although SRT does not support recovery from errors, the following features introduced by SRT for error detection [8] are important for recovery as well: (1) Replicating cached loads is problematic because memory loca- 
tions may be modified by an external agent (e.g., another processor during multiprocessor synchronization) between the time the leading thread loads a value and the time the trailing thread tries to load the same value. The two threads may diverge if the loads return different data. SRT allows only the leading thread to access the cache, and uses the Load Value Queue (LVQ) to hold the leading load values. The trailing thread loads from the LVQ instead of repeating the load from the cache. (2) The leading thread runs ahead of the trailing thread by a long slack (e.g., 256 instructions), and provides the leading branch outcomes to the trailing thread through the Branch Outcome Queue (BOQ). The slack and the use of branch outcomes hide the leading thread's memory latencies and branch mispredictions from the trailing thread, since by the time the trailing thread needs a load value or a branch outcome, the leading thread has already produced it. (3) By replicating register values but not memory values, SRT compares only stores and uncached loads, and not register values, of the two threads. Because an incorrect value caused by an error propagates through computations and is eventually consumed by a store, checking only stores suffices.

A recent paper [7] proposes hardware recovery using superscalar hardware without any SMT support. It advocates the natural way to achieve recovery by using superscalar's rollback ability. It does not use the LVQ and does not address the issues related to cached loads. It also claims that there is no need for any slack in superscalar. However, for an SRT using the LVQ for cached loads, we confirm SRT's results that zero slack causes trailing thread stalls and performance loss due to unavailable leading load values. Our results show that the unavailability is due largely to on-chip cache hit latencies, and that slack would have been needed in [7] if it had addressed cached loads.

We propose Simultaneously and Redundantly Threaded processors with Recovery (SRTR) to extend SRT to include recovery. Although systems using software recovery often employ hardware detection [3,11], software checkpointing incurs considerable performance cost even when there are no faults. Therefore, hardware recovery at a modest performance cost over detection is an attractive option, especially because hardware recovery permits the use of stock software. We identify the following key issues not addressed in [7]:

- Problem: A fundamental implication of the SRT detection scheme is that leading non-store instructions may commit before checking for errors, relying on the trailing thread to detect errors when the trailing stores complete. SRTR, on the other hand, must not allow any leading instruction to commit before checking since a faulty instruction cannot be undone once the instruction commits. Unless care is taken, leading instructions will stall at commit waiting for their trailing counterparts to complete and check. This will create pressure on the instruction window and physical registers, and degrade performance. Solution: To avoid leading instructions stalling at the commit point, SRTR exploits the time between completion and commit of a leading instruction. SRTR checks the results of a leading and the corresponding trailing instruction as soon as the trailing instruction completes, well before the leading instruction reaches the commit point. For the SPEC95 benchmarks, the complete to commit time averages at 29 cycles. This provides sufficient time for a trailing instruction to complete before the leading instruction reaches the commit point. To exploit the complete to 
commit time, the slack between the leading and trailing threads in SRTR must be short. At the same time, a slack that is too short would cause the trailing thread to stall due to unavailable branch outcomes and load values from the leading thread. To support an appropriately short slack, SRTR's leading thread provides the trailing thread with branch predictions instead of outcomes. Because the leading thread's branch predictions are available much earlier than the branch outcomes, and because a short slack is sufficient for hiding on-chip cache hit latencies, SRTR avoids trailing thread stalls even with a short slack. We show that high prediction accuracies and low off-chip miss rates in the base SMT enable SRTR to perform within 5\% of SRT when the recovery mechanisms of SRTR are disabled so that both schemes target only detection. In this experiment, SRTR uses a slack of 32, and SRT uses a slack of 256 as in [8].

- Problem: By the time a leading instruction reaches the commit point, its register value often has been written back to the physical register file. Because all instructions are checked in recovery, accessing the register file in order to perform the check will add substantial bandwidth pressure. Solution: SRTR uses a separate structure, the register value queue $(R V Q)$, to store register values and other information necessary for checking of instructions, avoiding bandwidth pressure on the register file.

- Problem: There is bandwidth pressure on the RVQ. Solution: We propose dependence-based checking elision $(D B C E)$ to reduce the number of checks, and thereby, the RVQ bandwidth demand. By reasoning that faults propagate through dependent instructions, DBCE exploits register (true) dependence chains so that only the last instruction in a chain uses the RVQ to check leading and trailing values. The chain's earlier instructions in both threads completely elide the RVQ. SRT can be viewed as taking such elision to the extreme by observing that stores are the last instructions in any register dependence chain, and checking only stores. However, SRT's chains are too long for SRTR because the leading thread cannot commit until the last instruction in the long chain checks. DBCE forms short chains by exploiting the abundant register dependencies in near-by instructions. Because of the short slack and short chains, the trailing chain's last instruction completes only a few cycles behind the leading chain's earlier instructions. Consequently, checking of the last instructions is usually done between the time the earliest leading instruction completes and the time it reaches the commit point. DBCE redundantly builds chains in both threads and checks its own functionality for faults. DBCE elides $35 \%$ of checks for the benchmarks we consider. The performance of SRTR is within $1 \%$ and $7 \%$ of SRT performance for SPEC95 integer and floating-point programs, respectively. SRTR without DBCE incurs about $18 \%$ performance loss when the number of RVQ ports is reduced from four (which is equivalent to an unlimited number) to two ports. With DBCE, a 2-ported RVQ performs within 2\% of a 4-ported RVQ.

SRTR is guaranteed to provide recovery from single transient errors except for the errors that affect the register file and the errors that fail to propagate through dependence chains, in which case it guarantees detection.

We review SRT in Section 2. We describe the SRTR scheme in Section 3 and DBCE in Section 4. In Section 5, we present experimental results. In Section 6, we discuss related work, and conclude in Section 7.

\section{Transient Fault Detection: Background}

SRT uses SMT's multithreaded execution to replicate an application into two communicating threads, one executing ahead of the other. Comparing the results of two redundant executions is the basic underlying scheme to detect transient faults in SRT. Because detection is based on replication, the extent to which the application is 
replicated is important. SRT formalizes this notion by defining the sphere of replication (SoR) [8], pointing out that (1) all computation and data within this sphere are replicated such that each thread uses its own copy, (2) data entering the SoR is independently read by the two threads using input replication, (3) data exiting the SoR from the two threads are compared using output comparison, and only one copy of the checked data exits. Because data outside the SoR is not replicated, other forms of protection such as ECC are needed outside the SoR.

Two possible SoRs defined in [8] are: processor and registers inside the SoR, with the cache hierarchy outside; and only the processor inside the SoR, with the registers and cache hierarchy outside. In the first SoR, leading and trailing thread values need to be compared only for stores and uncached loads, while other instructions can commit without comparing values. SRT uses a store buffer (StB) in which the leading thread places its committed store values and addresses. The trailing thread compares the StB entries against its own store values and addresses to determine whether an error has occurred. In the second SoR, all register values need to be checked. Checking registers requires large buffers with high bandwidth to avoid performance degradation.

As explained in Section 1, to handle cached loads, SRT uses the ECC-protected Load Value Queue (LVQ) in which the leading thread deposits its committed load values and addresses. The trailing thread matches its addresses to those in the LVQ, and obtains the load value from the LVQ instead of repeating the load from the memory hierarchy. The Active Load Address Buffer proposed in [8] is an alternative for the LVQ that also addresses this problem. We use the LVQ because it is simpler.

A key optimization in SRT is that the leading thread runs ahead of the trailing thread by an amount called the slack (e.g., the slack may be 256 instructions). In addition, the leading thread provides its branch outcomes via the branch outcome queue (BOQ) to the trailing thread. The slack and the communication of branch outcomes hide the leading thread's memory latencies and avoids branch mispredictions from the trailing thread. Due to the slack, by the time the trailing thread needs a load value or branch outcome, the leading thread has already produced it.

SRT assumes that uncached accesses are performed non-speculatively. SRT synchronizes uncached accesses from the leading and trailing threads, compares the addresses and replicates the load data. For replication of instructions, SRT assumes that code does not modify itself, but points out that self-modifying code in regular SMTs already requires thread synchronization and cache coherence which can be extended to provide consistency between the leading and trailing threads. For input replication of external interrupts, SRT suggests forcing the threads to the same execution point and then delivering the interrupt synchronously to both threads.

\section{Transient Fault Recovery}

We propose Simultaneously and Redundantly Threaded processors with Recovery (SRTR) that enhances SRT 
to include transient fault recovery. A natural way to extend SRT to achieve recovery is to use the rollback ability of modern pipelines, which is provided to support precise interrupts and speculative execution [7]. Because transient faults do not persist, rolling back up to and including the offending instruction and re-executing guarantees forward progress.

In SRT, however, leading non-store instructions may commit before checking for faults, relying on the trailing instruction to check either store values in the first SoR or register values in the second SoR. SRTR, on the other hand, must not allow any instruction to commit before it is checked for faults. As much as possible, trailing instructions must complete and results must be compared before the leading instructions reach the commit point so that leading instructions do not stall at commit. Therefore, the slack between the threads cannot be long. At the same time, a near-zero slack would cause the trailing thread to stall due to unavailable branch outcomes and load values from the leading thread. To support a short slack, SRTR's leading thread provides the trailing thread with branch predictions instead of outcomes, as done by SRT. Accordingly, SRTR counts slack in terms of fetched speculative instructions, while SRT counts slack in terms of committed instructions.

SRT uses committed values for branch outcomes, load addresses and values, and store addresses and values. Consequently, the StB, LVQ, and BOQ are simple queues that are not affected by mispredictions. Because SRTR compares speculative values of the leading and trailing threads, it needs to handle the effects of mispredictions on these structures.

SRTR uses the SoR that includes the register file. Like SRT, we assume an out-of-order, SMT pipeline [15]. The pipeline places instructions from all threads in a single issue quеие. Instructions wait in this queue until source operands become available, enabling out-of-order issue. Apart from the issue queue, each thread's instructions are also held in the thread's private active list $(A L)$. When an instruction is issued and removed from the issue queue, the instruction stays in its AL. Instructions commit from the AL in program order, enabling precise interrupts.We illustrate SRTR's SoR and SRTR's additions to SMT in Figure 1.

\subsection{Keeping speculative leading and trailing instructions synchronized}

For every branch prediction, the leading thread places the predicted PC value in the prediction queue (predQ). This queue is similar to the BOQ except that it holds predictions instead of outcomes. The predQ is emptied in queue-order by the trailing thread. Using the predQ, the two threads fetch essentially the same instructions without diverging.

Because the ALs hold the instructions in predicted program order and because the two threads communicate via the predQ, corresponding leading and trailing instructions occupy the same positions in their respective ALs. Thus, they can be easily located for checking. Note that corresponding leading and trailing instructions may enter their ALs at different times, and become ready to commit at different times. However, we will be 


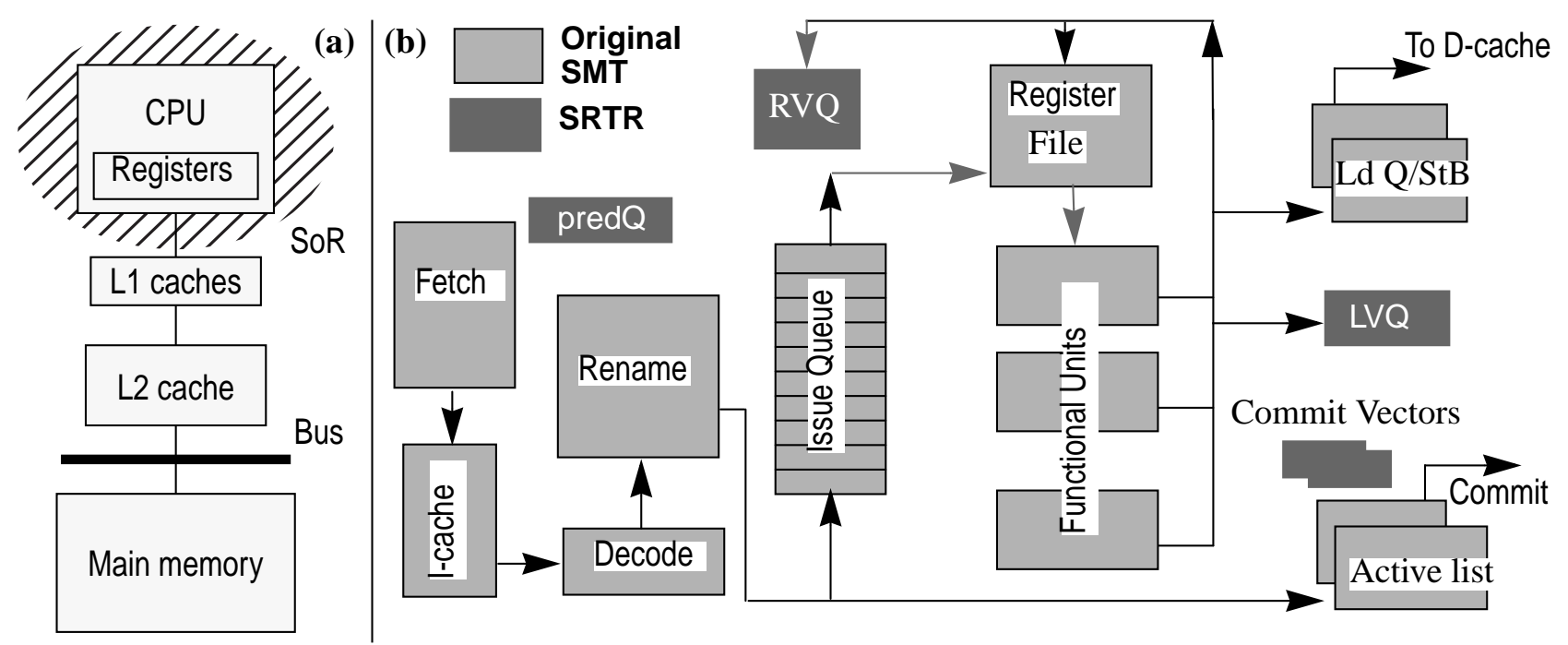

FIGURE 1: (a) SRTR's Sphere of Replication. (b) SRTR's additions to SMT.

able to use the fact that the instructions occupy the same position in their ALs to keep the implementation simple.

Due to the slack, the leading and trailing threads resolve their branches at different times. Upon detecting a misprediction in the leading thread, the leading thread cleans up the predQ, preventing the trailing thread from using mispredicted entries placed earlier in the predQ. There are two possibilities for the timing of events related to a misprediction: (1) The leading branch resolves after the trailing thread has already used the corresponding predQ entry, or (2) the leading branch resolves before the entry is used by the trailing thread.

Possibility 1 implies that the trailing AL position corresponding to the leading branch's AL pointer is valid and contains the trailing branch. There are mispredicted trailing instructions in the trailing AL. The leading thread then squashes the mispredicted instructions in the trailing AL, and the existing predQ entries which contain fetch PCs from the incorrect path. Because the leading and trailing ALs are identical, the leading branch can use its own AL pointer to squash the trailing AL. Possibility 2 implies that the trailing AL position corresponding to the leading branch's AL pointer is beyond the tail of the trailing thread AL. In this case, the leading branch squashes all predQ entries later than its own predQ entry and places the branch outcome in the predQ to be used by the trailing thread later. To prevent errors from causing incorrect squashing, AL pointers are protected by ECC.

Although the leading thread rolls back the predQ and the ALs of both threads upon a misprediction, the trailing thread still checks the leading branch's outcome against the trailing branch's outcome. The rollback is an optimistic action to reduce the number of mispredicted trailing instructions, assuming that the leading thread is fault-free. If the leading thread's misprediction is incorrectly flagged due to a fault, the trailing branch's check triggers a rollback. We discuss the details of checking in Section 3.3. 


\subsection{Modifying the LVQ}

SRT uses a strict queue-ordering for the LVQ, i.e., the leading thread inserts committed load values and addresses at the tail, and the trailing thread empties the load values and addresses from the head of the queue. SRTR modifies SRT's LVQ to operate on speculative cached loads, and therefore, cannot maintain the strict queue order of SRT. To keep the LVQ as compact as possible, we use a table, called the shadow active list $(S A L)$, to hold pointers to LVQ entries. The SAL mirrors the AL in length but is much narrower than the LVQ, and instructions can use the AL pointer to access their information in the SAL. The SAL is also helpful in checking register values as explained later.

A leading load allocates an LVQ entry when the load enters the AL, and places a pointer to the entry in the SAL. Because loads enter the AL in (speculative) program order, LVQ entries are allocated in the same order, simplifying misprediction handling as explained at the end of the section. Upon issue, the leading load obtains its LVQ pointer from the SAL and places its load value and address in the LVQ. The trailing load, when it is issued, also obtains the LVQ pointer from the SAL, and it compares its address with the leading load address stored in the LVQ, as done in SRT. On a match, the trailing load obtains the leading load value from the LVQ. A mismatch of the addresses flags a rollback (as explained in Section 3.3) with three possibilities: (1) the address register value produced by a previous instruction is faulty and the faulty instruction will cause rollback to be initiated upon being checked; (2) the address computation of the load is faulty and the load instruction will cause rollback to be initiated; (3) the previous instruction was checked and committed and the address register has been corrupted since. Because the register file is inside the SoR, SRTR flags an error but cannot recover in this case without protecting the register file with ECC.

Even though leading instructions are fetched and placed ahead of the corresponding trailing instructions in the issue queue, it is possible that a trailing load is issued before the leading load. A possible solution is to place the premature trailing load's address in the empty LVQ entry. Upon arriving at the LVQ, the leading load compares the addresses and satisfies the pending trailing load. This solution naturally extends to the case where a trailing load issues after the leading load, but finds the LVQ entry empty due to the leading load missing in the cache. Note that the LVQ is ECC-protected and so values stored in it are not vulnerable to faults.

An LVQ entry is relinquished in queue order after the trailing instruction reads the entry. Rollback of the SAL upon a leading branch misprediction is done in parallel with the clean-up of the AL. To facilitate the rollback of the LVQ, branches place the LVQ tail pointer in the SAL at the time they enter the AL. Because the LVQ is in (speculative) program order, the LVQ tail pointer points to the LVQ entry to which the LVQ needs to be rolled back, if the branch mispredicts. A mispredicted branch's AL pointer locates the LVQ tail pointer in the SAL, and the LVQ is rolled back to the pointer. Like the predQ's rollback, the LVQ's rollback is also an optimistic action and the leading branch is checked to confirm the misprediction. 
FIGURE 2: SRTR pipeline with fault-check stage.
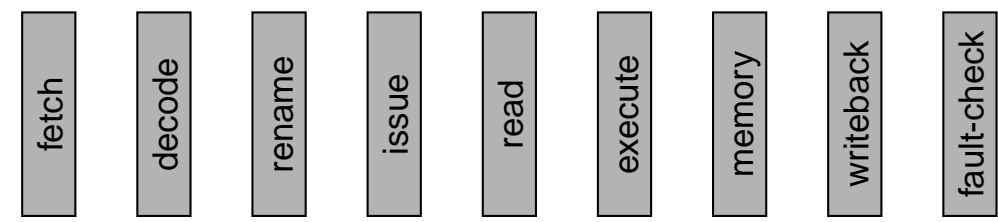

\subsection{Checking leading and trailing instructions}

SRTR checks the leading and trailing instructions as soon as the trailing instruction completes. Register values often have been written back to the physical register file by the time the check is performed. To address this issue, SRTR uses a separate structure, the register value queue ( $R V Q)$ to store register values for checking, avoiding bandwidth pressure on the register file (Figure 1(b)). In this section, we assume that all the instructions including branches but excluding loads and stores use the RVQ to check. We assume that the RVQ can provide the required bandwidth. We address the bandwidth pressure on the RVQ in Section 4.

Because the trailing instructions need to locate the leading counterpart's value in the RVQ, the leading instruction allocates an RVQ entry at the time of entering the AL, and places a pointer to the entry in the SAL for the trailing instruction. After the leading instruction writes its result back, it enters the fault-check stage. In the fault-check stage, the leading instruction puts its value (for branches, the next PC, the prediction and the outcome are all part of the value) in the RVQ using the pointer it obtains from the SAL. The instruction then waits in the AL to commit or squash due to faults or mispredictions. Because the fault check stage is after writeback, the fault check stage does not affect branch misprediction penalty, or the number of bypass paths. Figure 2 illustrates this point.

The trailing instructions also use the SAL to obtain their RVQ pointers and find their leading counterparts' values. While it is likely that the leading instruction reaches the fault check stage before the trailing instruction, out-of-order pipelines may reverse the order. To handle such reversals, if the trailing instruction finds the RVQ entry of its leading counterpart to be empty, it places its own value. When the leading instruction reaches the fault-check stage, it finds the value and performs the check. A full/empty bit in the RVQ is used to indicate whether or not the RVQ entry contains leading or trailing values. The full/empty bits need to be ECC-protected to prevent corrupted full/empty bits from causing leading and trailing instruction pairs to wait forever. An RVQ entry is relinquished in queue order after the checking is done. Branch misprediction clean-up of the RVQ uses the SAL in the same way as for LVQ clean-up.

SRTR places completed leading store values and addresses in the StB. Completed trailing stores check the values and addresses. Address mismatches in the StB are handled similar to those in the LVQ.

Leading and trailing values are compared either in the RVQ or in the StB, and if the check succeeds the commit vector $(\mathrm{CV})$ entries corresponding to the leading and trailing instructions are set to the checked-ok state. 
Indexed by AL pointers, the CVs are parallel to the ALs (Figure 1(b)). As instructions are placed in the AL, their CV entries are set to the not-checked-yet state. Due to the slack, leading instructions commit ahead of their trailing counterparts from their respective ALs. If the state is not-checked-yet, the instruction is stalled at commit until it is checked. If a check fails, the $\mathrm{CV}$ entries of the leading and trailing instructions are set to the failed-check state.

A leading instruction commits only if its CV entry and the CV entry of its trailing counterpart are in the checked-ok state. When a failed-check entry reaches the head of the leading AL, all later instructions are squashed. The leading thread waits until the trailing thread's corresponding entry reaches the head of the trailing AL before restarting both threads at the offending instruction. Because there is a time gap between the setting and the reading of the $\mathrm{CV}$ and between the committing of leading and trailing counterparts, the $\mathrm{CV}$ is protected by ECC to prevent faults from corrupting it in the time gap.

There are faults from which SRTR cannot recover: After a register value is written back and the instruction producing the value has committed, if a fault corrupts the register, then the fact that leading and trailing instructions use different physical registers will allow us to detect the error on the next use of the register value. However, SRTR cannot recover from this error. To avoid this loss of recovery, one solution is to provide ECC on the register file.

\section{Reducing Bandwidth demand on the Register Value Queue}

The RVQ needs to allow as many writes or reads of register values per cycle as the number of leading and trailing instructions completing in one cycle. Because the RVQ has as many entries as in-flight leading instructions (around 120 64-bit values, totaling to $1 \mathrm{~KB}$ ), providing multiple ports to support high bandwidth (e.g., four 64bit values per cycle) may be unacceptable.

We propose dependence-based checking elision $(D B C E)$ to reduce the number of instructions accessing the RVQ. To keep the implementation simple, we use only simple dependence chains such that each instruction in a chain has at most one parent and one child (instead of maintaining the full dependence graph). By reasoning that faults propagate through dependent instructions, DBCE exploits register (true) dependence chains so that only the last instruction in a chain uses the RVQ to check leading and trailing values, as shown in Figure 3(a). The chain's earlier instructions in both threads completely elide the RVQ. If the last instruction check succeeds, it signals the previous instructions in the chain that they may commit; if the check fails, all the instructions in the chain are marked as having failed and the earliest instruction in the chain triggers a rollback. A key feature of DBCE is that both leading and trailing instructions redundantly go through the same dependence chain formation and checking-elision decisions, allowing DBCE to check its own functionality for faults. 


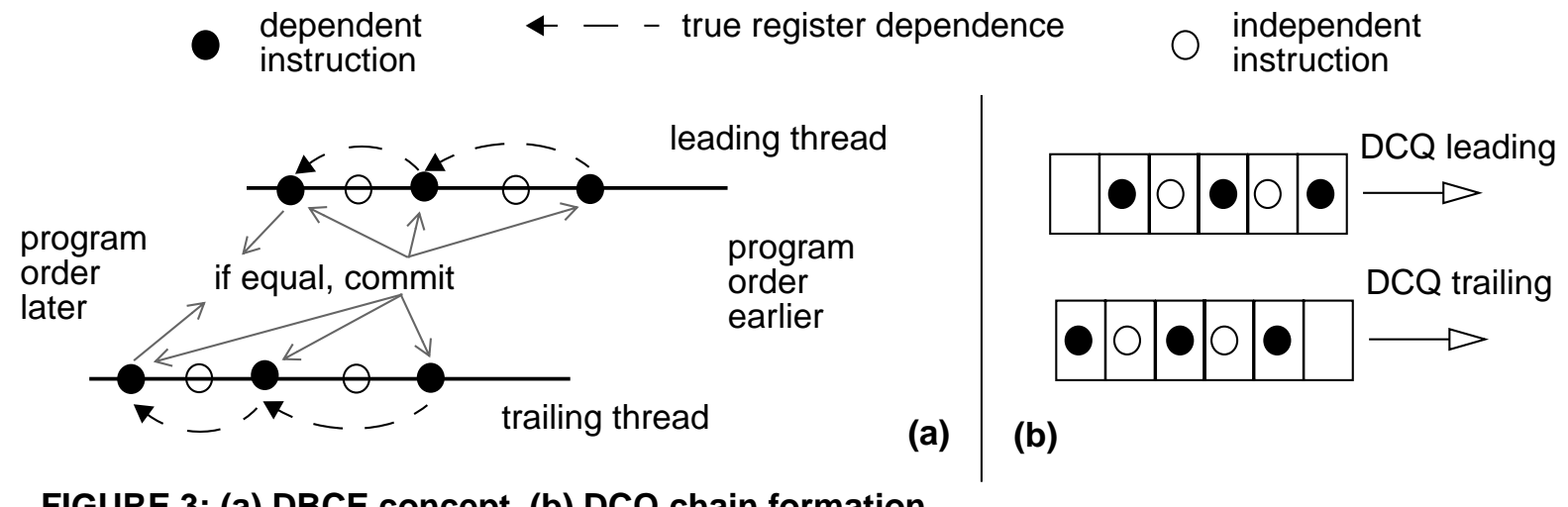

FIGURE 3: (a) DBCE concept. (b) DCQ chain formation.

If the last instruction of a chain is further in the instruction stream and completes much later than the other instructions, the chain's earlier instructions will stall at commit. To avoid this situation, DBCE forms short dependence chains (e.g., 3-4 instructions) by exploiting the abundant register dependencies in near-by instructions. If DBCE's chains are $m$ instructions long, DBCE checks only one out of $m$ instructions, reducing the bandwidth by a factor of $m$. Because of short slack and short chains, the trailing chain's last instruction completes just a few cycles behind the leading chain's earlier instructions. Consequently, checking of the last instructions is usually done between the time the earliest leading instruction completes and the time it reaches the commit point.

Because leading loads deposit their values in the LVQ for the trailing loads, there is no notion of eliding of checking for load values, and hence loads are not included in the chains. Stores are checked in the StB and do not use the RVQ. Therefore, stores are not included in the chains as well. Because branches do not produce register values, branches cannot be in the middle of a chain. Branches may be at the end of chains and in that case they themselves cannot elide checking but they help elide checking for the instructions preceding in their chain. If chains are allowed to cross branches, mispredictions will require clean-up of the chains to deal with chains whose later instructions have been squashed due to an intervening branch misprediction. To avoid such clean-ups, DBCE disallows chains from crossing branches.

Exploiting dependence chains consists of (1) identifying dependence chains in the leading thread and the corresponding chains in the trailing thread, and tagging each chain with a unique tag, (2) identifying the instructions in the leading and trailing threads performing the check, (3) preventing the rest of the instructions (leading and trailing) in the chain from performing the check, and (4) notifying the non-checking instructions in the chain after the check is performed.

\subsection{Forming dependence chains}

To identify dependence chains, DBCE uses the dependence chain queue (DCQ), which holds information about renamed instructions that were fetched in the last few cycles (e.g., 1-2 cycles). The instructions are kept 
in the instruction fetch order. Each entry in the queue holds the destination physical register and the AL pointer of an instruction, a tag which identifies the chain to which the instruction belongs, and a flag to indicate if the instruction already has a dependent instruction in its dependence chain. The AL pointer of the first instruction in a chain is used as the chain's tag.

Upon entering the DCQ, an instruction associatively searches the DCQ using its source physical register numbers, matching them against destination physical register numbers of instructions in the DCQ. If there is no match on any source register, or if all the matching instructions already have their flags set indicating that those instructions already have children, there is no live chain to which the current instruction can belong; then the instruction uses its own AL pointer as its tag, and it clears its flag to start a new chain. Branches cannot start a chain, and they are removed from the DCQ if they cannot join a live chain. If there is a matching entry with a clear flag, the current instruction adds itself to the matching entry's chain by setting the matching entry's flag and obtaining the matching entry's AL pointer and tag. It clears its flag to allow additional instructions to join the chain. If two sources of an instruction match entries with clear flags, the current instruction adds itself to one of the two chains (the one corresponding to the first source).

The leading and trailing instructions form chains independently. Because there are no dependencies between the two threads, the DCQ can hold the two threads simultaneously. However, because fetching of leading and trailing instructions is interleaved, care must be taken to ensure that the DCQ will form identical dependence chains in the leading and trailing threads. The chains formed may be different if fetch brings a number of leading instructions, switches and brings a smaller number of trailing instructions before switching back to the leading thread. The larger number of leading instructions may cause longer chains to be formed than the fewer trailing instructions.

A simple solution is to have the leading and trailing threads each occupy half the DCQ, as shown in Figure 3(b). Every cycle either leading or trailing thread instructions reach the DCQ. The DCQ evicts the oldest entries of the same thread to make room for the new instructions. Before evicting the oldest entries, the DCQ terminates the chains originating at the entries, ensuring that the chains stay short and span at most as many cycles as the DCQ depth. The instructions in the terminated chains are recorded in the check table for later use. The AL pointer of each of the oldest entries is used to search the DCQ tags, and the matching entries are all the instructions in the chain originating at the oldest entry. Although the number of oldest entries may be as large as the fetch width, requiring as many parallel searches of the DCQ, the DCQ's small size keeps this parallel search manageable (e.g., 8-way search of 16 entries).

The matching DCQ entry that has a clear flag is the last instruction in the oldest entry's chain. The chain's nonlast instructions use their AL pointers to index into the check table. There, they set the not-check bits to indicate that they elide checking, and they update their last-instruction fields with the last instruction's AL pointer. 
The chain's last instruction keeps its not-check bit clear and updates its last-instruction field with its own AL pointer. Thus, the check table records the chain, identifying the chain's last instruction as needing to check and the rest of the instructions as eliding checking. The check table also associates the eliding instructions with the last instruction whose check covers them.

Operating on renamed instructions guarantees that matching source registers with previous destinations without checking for multiple matches in the DCQ is correct. Also, the DCQ implements a subset of the functionality of renaming and bypass. While it may be possible to use the existing renaming and bypass logic, we describe the DCQ separately for clarity and to avoid assuming details of rename and bypass.

\subsection{Using the dependence chains}

When a leading or trailing instruction reaches writeback, the instruction uses its AL pointer to index into the check table and obtain the AL pointer of the last instruction in its chain. The instruction compares the leading and trailing not-check bits in the check table entry. If the bits do not match (indicating a mismatch between the leading and trailing chains caused by an error), the CV entries of the last instructions in both chains are set to failed-check. Otherwise, if the not-check bit is set, the instruction elides checking and waits in the AL to commit, holding its last instruction AL pointer. If the not-check bit is clear, the leading instruction places its value in the RVQ for the trailing instruction. Note that the AL pointers carried by the instructions are ECC protected. This allows the leading and trailing instructions to access the check table independently without checking against each other's AL pointers.

Later, the trailing instruction indexes into the check table and compares the not-check bits as above. If both the leading and trailing not-check bits are clear, the trailing instruction obtains the leading instruction's value from the RVQ, and it compares the value against its own. If the check succeeds, the trailing AL pointer is used to mark the CV entries of both leading and trailing instructions as checked-ok. If the check fails, the CV entries are marked as failed-check. In a chain, only the last-instruction's CV entries are marked, and the rest of the instructions' CV entries remain in the not-checked-yet state. If the trailing instructions reach the RVQ first, then the role of the leading and trailing instructions reverse in the above.

When a leading instruction reaches the head of the AL, it compares its last-instruction's AL pointer to that of its trailing counterpart. On a match, the leading instruction uses its last-instruction's AL pointer to probe the leading and trailing CV entries. Depending on the CV entries, the instruction waits (if the CV entries are notchecked-yet), commits (if the CV entries are checked-ok), or squashes (if the CV entries are failed-check); squashing also occurs on a mismatch. When a trailing instruction reaches the head of its AL, if the leading thread has already committed, the CV entry of the trailing thread is guaranteed to be checked-ok and the trailing instruction will commit. 


\begin{tabular}{|l||l|}
\hline Component & Description \\
\hline \hline Processor & 8-way out-of-order issue,128-entry issue queue \\
\hline $\begin{array}{l}\text { Branch } \\
\text { prediction }\end{array}$ & $\begin{array}{l}\text { hybrid 8K-entry bimodal, 8K-entry gshare, 8K2-bit selector } \\
\text { 16-entry RAS, 4-way1K BTB (10-cycle misprediction penalty) }\end{array}$ \\
\hline L1 I- and D-cache & 64KB, 32-byte blocks, 4-way, 2-cycle hit, lock-up free \\
\hline L2 unified cache & 1 Mbyte,64-byte blocks, 4-way, 12-cycle hit, pipelined \\
\hline Main memory & Infinite capacity, 100 cycle latency \\
\hline
\end{tabular}

Table 1: Hardware parameters for base system.

Upon instruction commit, the check table entry corresponding to the committing instruction's AL pointer are cleared. On an instruction squash, the check table entries corresponding to the squashed AL pointers are invalidated. Because the DCQ holds later instructions down the stream from the squashing instruction, all the DCQ entries from the squashing thread are discarded on a squash.

The DCQ has to support high bandwidth of instructions every cycle. Because the DCQ holds a small number of instructions, it can be implemented to support high bandwidth (e.g., 8-16 instructions each requiring one 8bit destination register number, three 8-bit AL pointers, and a few bits for a flag, totalling to about 80 bytes). Providing high bandwidth to the RVQ which is kilobytes in size is harder, for the same reason that rename tables are widely multiported but D-caches are only dual-ported. The check table is a high bandwidth structure because every leading and trailing instruction accesses it. Because the check table holds only two AL pointers and a flag per entry, multi-porting it is easier than multi-porting the much-larger RVQ.

\section{Experimental Results}

We modified the Simplescalar out-of-order simulator [2] to model SMT and SRT. Table 1 shows the base system configuration parameters used throughout the experiments. The front-end of our base pipeline is long to account for SMT and deep pipelines corresponding to high clock speeds. Like SRT, we approximate a highbandwidth trace cache by fetching past three branches, and at most eight instructions, per cycle. Table 2 presents the SPEC95 benchmarks and their inputs used in this study. In the interest of space, we show results for the subset of the SPEC95 applications, which are representative of our results over the entire SPEC95 suite. We run the benchmarks to completion, or stop at 1 billion instructions ${ }^{1}$ in the interest of simulation time.

We present results in the absence of faults in order to study the performance cost of SRTR over SRT. In the presence of faults, SRTR can recover but SRT will stop as soon as it detects a fault. Therefore, a comparison of performance is not possible when faults occur. In addition, faults are expected to be rare such that the overall performance will be determined by fault-free behavior.

1. For these cases, the cumulative IPC (instructions per cycle) stopped changing over the last 800 million instructions. 


\begin{tabular}{|l||l|r|r|l||l|r|r|}
\hline Benchmark & Input & \#instrs x 10 & base IPC & Benchmark & Input & \#instrs x 10 & base IPC \\
\hline \hline go & train & 600 & 1.17 & compress & train & 40 & 2.16 \\
\hline lisp & test & 1000 & 1.63 & swim & test & 780 & 2.53 \\
\hline gcc & test & 1000 & 1.28 & applu & train & 680 & 2.93 \\
\hline perl & jumble & 1000 & 1.90 & fpppp & train & 510 & 0.59 \\
\hline ijpeg & vigo & 1000 & 2.58 & su2cor & test & 1000 & 2.18 \\
\hline vortex & train & 1000 & 2.12 & hydro2d & test & 1000 & 1.94 \\
\hline m88ksim & test & 500 & 2.89 & tomcatv & test & 1000 & 2.69 \\
\hline
\end{tabular}

Table 2: Benchmarks and inputs.

Because the basic scheme used in SRTR for detection is different from that used in SRT, we start by comparing the SRTR detection scheme (without recovery) against SRT. We refer to the detection scheme implemented in SRTR as SRTRd. SRTRd uses a short slack and it communicates branch predictions between the leading and trailing threads, while SRT uses a long slack and communicates branch outcomes. In the same experiment, we also show the performance impact of near-zero slack. Then, we report the average time gap between complete to commit, and average memory latencies in the base SMT. These parameters determine the constraints on SRTR's slack which needs to be shorter than the average complete to commit gap to avoid leading thread stalls at commit, but long enough to avoid trailing thread stalls due to empty LVQ. We then compare SRTR (providing recovery) using a high-bandwidth RVQ, to SRTRd and SRT. This comparison gives the performance cost of recovery over detection. We show the impact of the RVQ size on SRTR's performance. Finally, we show the bandwidth reduction achieved by DBCE while maintaining the same performance as the high-bandwidth RVQ.

\subsection{SRT vs. SRTRd}

In Figure 4, we compare SRTRd and SRT. We show the performance normalized to the base SMT executing only the standard program. We use a slack of 256 and a 256-entry BOQ, 256-entry LVQ and 256-entry StB for SRT, exceeding the sizes for the best performance reported by the SRT paper [8]. For SRTRd, we use predQ/ LVQ/StB sizes of 128/128/128 for a slack of $128,80 / 96 / 80$ for a slack of $64,48 / 96 / 48$ for a slack of 32 , and 18/ $96 / 18$ for a slack of 2 . The purpose of this experiment is to demonstrate the effect of using a short slack and communicating branch predictions between the leading and trailing threads, versus using a long slack and communicating branch outcomes. We do not want the queues filling up to interfere with this comparison. Therefore, we keep the sizes of SRTRd's queues appropriately large for each slack value. It is important to note that SRTR needs a short slack to avoid leading instructions stalling at commit while waiting for trailing instructions to complete and check. This effect does not exist in SRTRd, which performs well with higher values of slack.

From Figure 4, the performance of SRT across integer and floating point (FP) programs is between $2 \%$ to $45 \%$ worse than the base SMT. These numbers are in line with the SRT paper [8]. On average, SRT is $21 \%$ worse 


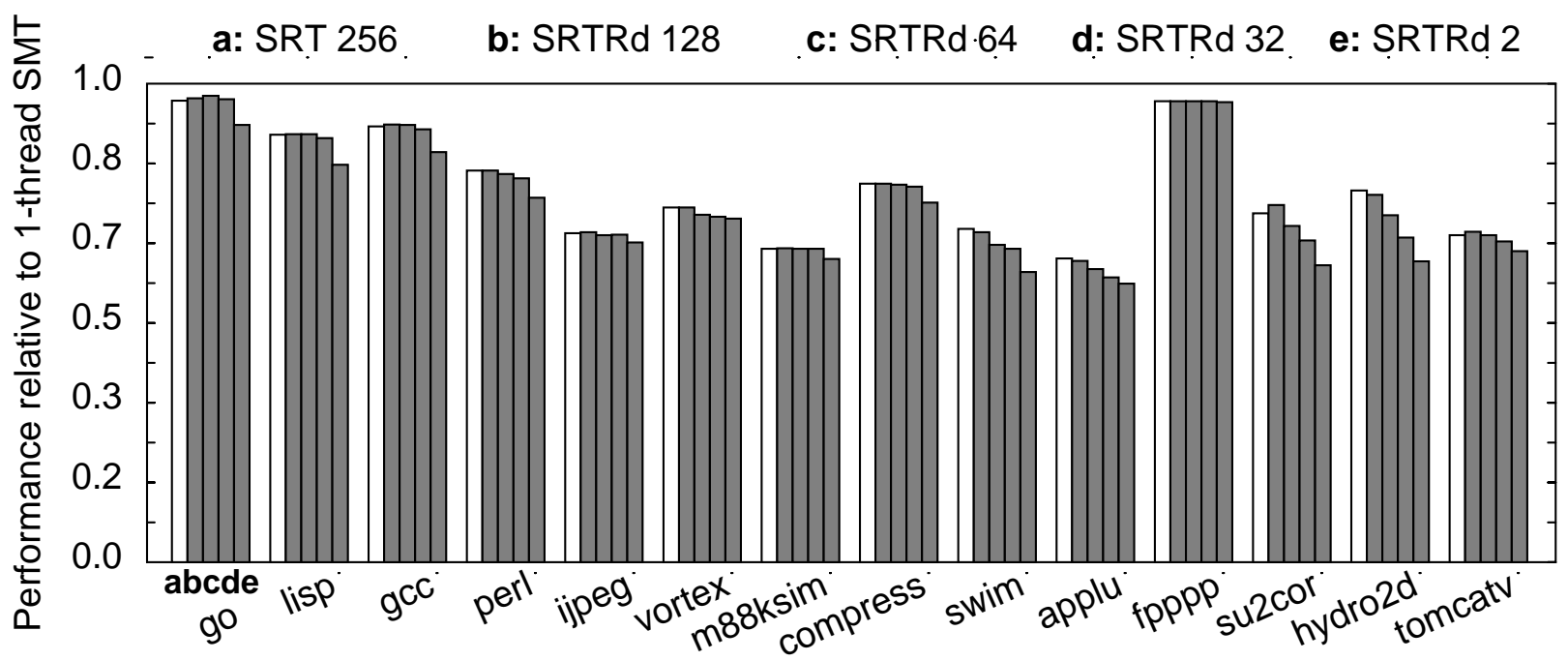

FIGURE 4: SRT vs. SRTR detection.

than SMT for the integer programs, and 27\% worse than SMT for the FP programs. In general, programs which exhibit enough ILP to saturate the processor resources in the base SMT incur higher performance loss due to replication in SRT (and SRTRd).

It can be seen that while a short slack of 64 or 32 performs close to a slack of 256, a near-zero slack of 2 incurs considerable performance loss for many programs. For the integer programs, SRTRd performs, on average, as well as SRT for slacks of 128 and 64, and within $1 \%$ of SRT for a slack of 32, showing that communicating branch predictions works as well as outcomes. For a slack of 2, STRTd performs about 5\%, on average, worse than SRT, with lisp incurring a 10\% performance loss. This loss is mainly due to unavailable load values in the LVQ (explained in Section 5.2). For the FP programs, SRTRd performs as well as SRT for a slack of 128, and within 3\% and 5\% of SRT for a slack of 64 and 32, respectively. For a slack of 2, SRTRd performs about 8\%, on average, worse than SRT, with hydro2d incurring a 26\% performance loss.

\subsection{Constraints on SRTR's Slack}

While SRTRd performs well with a large slack, recovery will require a shorter slack as discussed earlier. In this section, we explain why a short slack suffices, and how short the slack may be and still not impact performance. For SRT, SRTRd, and SRTR (providing recovery), the slack needs to be long enough to hide the leading thread's average memory latency from the trailing thread. However, SRTR's slack needs to be short enough so that trailing instructions can complete and check before the leading counterparts reach the commit point. Hence, SRTR's slack needs to be longer than the memory latency but shorter than the complete to commit time.

In Table 3, we tabulate the average number of cycles between complete and commit and the average memory latency for the base SMT. We compute the average memory latency as L1 hit time + L1 miss rate * L1 miss 


\begin{tabular}{|l||l|l|l||l|l|}
\hline Benchmark & $\begin{array}{l}\text { Av. memory } \\
\text { latency }\end{array}$ & $\begin{array}{l}\text { \#Av. complete } \\
\text { to commit time }\end{array}$ & Benchmark & $\begin{array}{l}\text { Av. memory } \\
\text { latency }\end{array}$ & $\begin{array}{l}\text { Av. complete to } \\
\text { commit time }\end{array}$ \\
\hline \hline go & 2.02 & 15.5 & compress & 2.89 & 26.5 \\
\hline lisp & 2.0 & 22.8 & swim & 3.36 & 39.5 \\
\hline gcc & 2.15 & 20.5 & applu & 3.64 & 34.3 \\
\hline perl & 2.22 & 27.3 & fpppp & 2.0 & 20.6 \\
\hline ijpeg & 2.15 & 27.4 & su2cor & 3.83 & 40.1 \\
\hline vortex & 2.15 & 39.4 & hydro2d & 5.80 & 45.4 \\
\hline m88ksim & 2.01 & 25.4 & tomcatv & 2.01 & 31.3 \\
\hline
\end{tabular}

Table 3: Slack constraints.

penalty + L2 miss rate * L2 miss penalty. This latency is the impact of the leading thread's load latency on the trailing thread assuming the worst case where the latency is completely exposed in the trailing thread. We see that due to good cache performance, the average memory latency is close to the hit time suggesting that the slack primarily needs to hide on-chip cache hit latencies. In general, the FP programs have a higher memory latency explaining their worse performance with shorter slacks. For instance, hydro $2 d$ has a long average memory latency, and performs poorly with a slack of 2 .

For all the programs, the gap between the average complete to commit time and the average memory latency is large enough to allow a slack longer than the average memory latency but shorter than the average complete to commit time. Even for memory-intensive applications which may have higher miss rates than our benchmarks, the gap is likely to be large enough to allow a reasonable slack. Note that slack is counted in number of instructions by which the leading thread is ahead, and the numbers in Table 3 are numbers of cycles. Because fetch can obtain up to 8 instructions per cycle, a slack of 32 is equivalent to 4 cycles.

\subsection{SRTR recovery}

The average complete to commit times in Table 3 suggest a range for appropriate slack values. To select an acceptable value for the slack, it is important to note that the complete to commit time of individual instructions vary quite widely. For instance, lisp, compress and tomcatv have 40\%, 50\%, and 40\%, respectively, instructions whose complete to commit times are fewer than 10 cycles. Therefore, a long slack may cause many leading instructions to stall at commit waiting for their trailing counterparts to complete and perform checking. It is thus important to select a slack value which accommodates the majority of the instructions.

In Figure 5, we compare SRT using a slack of 256 to SRTR (providing recovery) varying the slack for SRTR as $80,64,32$, and 2. Because SRTRd using a slack of 128 performs as well as SRT, we do not show SRTRd in this graph. To isolate the effect of the slack, we use a bandwidth-unlimited (i.e., 8 ports), 128-entry RVQ (we vary the RVQ size later). We use a 256-entry BOQ, 256-entry LVQ and 256-entry StB for SRT. For SRTR, we use predQ/LVQ/StB sizes of 128/128/128 for a slack of $80,80 / 96 / 80$ for a slack of $64,48 / 96 / 48$ for a slack of 32 , and 18/96/18 for a slack of 2. As in Section 5.1, we show performance normalized to the base SMT executing 


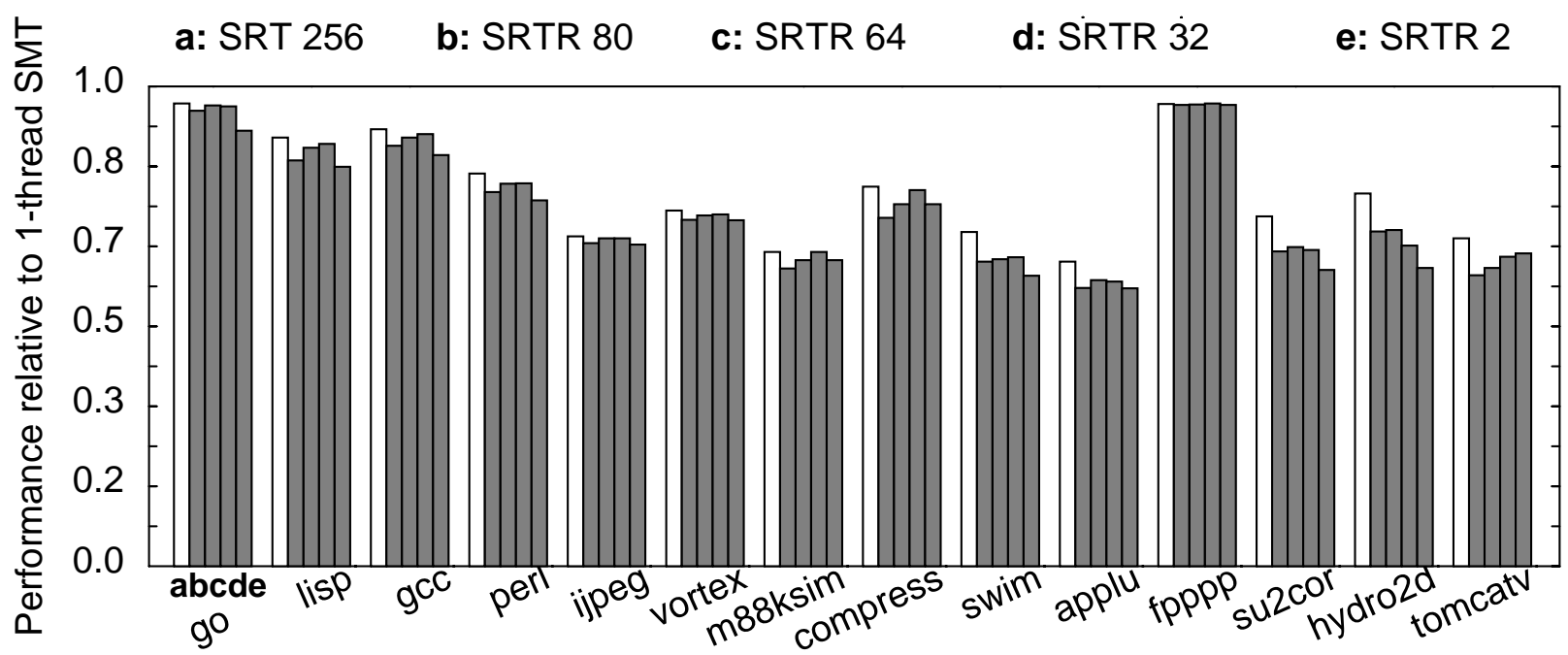

FIGURE 5: SRTR recovery.

only the standard program.

It can be seen that SRTR's average performance peaks at a slack of 32. For the integer programs, SRTR using a slack of 64 and 32 performs, on average, 3\% and 1\% worse than SRT. For the FP programs, SRTR performs, on average, 7\% worse than SRT for both a slack of 64 and 32. As expected, decreasing the slack to 2 causes performance degradation. Increasing the slack to 80 also causes performance degradation. SRTR using a slack of 80 performs 5\% and 9\%, on average, worse than SRT for the integer and FP programs, respectively. The main reason is that a slack of 80 makes the leading thread stall at commit, putting pressure on the instruction window. Thus, using a slack of 32 seems to be the best choice for these benchmarks.

\subsection{RVQ size}

In the experiment reported next, we measure the impact of varying the RVQ size on the performance of SRTR. RVQ entries are allocated as leading instructions enter the AL and freed in queue-order as the trailing counterpart obtains the RVQ value. Hence, the RVQ size depends on the issue queue size and the slack. In Figure 6, we compare SRT using a slack of 256 to SRTR using a slack of 32 (which was identified as the best value in the last section) and predQ/LVQ/StB sizes of 48/96/48, but varying the RVQ size as 128, 96, 80, and 64 entries. As before, we show performance normalized to the base SMT executing only the standard program.

It can be seen that an RVQ size of 80 entries works as well as 128 entries for all the programs. With 64 entries, while most programs experience no degradation, a few programs like gcc, compress and su2cor incur a small performance loss while ijpeg, applu, hydr2d and tomcatv slow down considerably. For these benchmarks, an RVQ size of 80 entries seems appropriate and achieves the same performance as a 128-entry RVQ.

\subsection{DBCE}

In this section, we show the effectiveness of DBCE in reducing the bandwidth demand on the RVQ. We mea- 


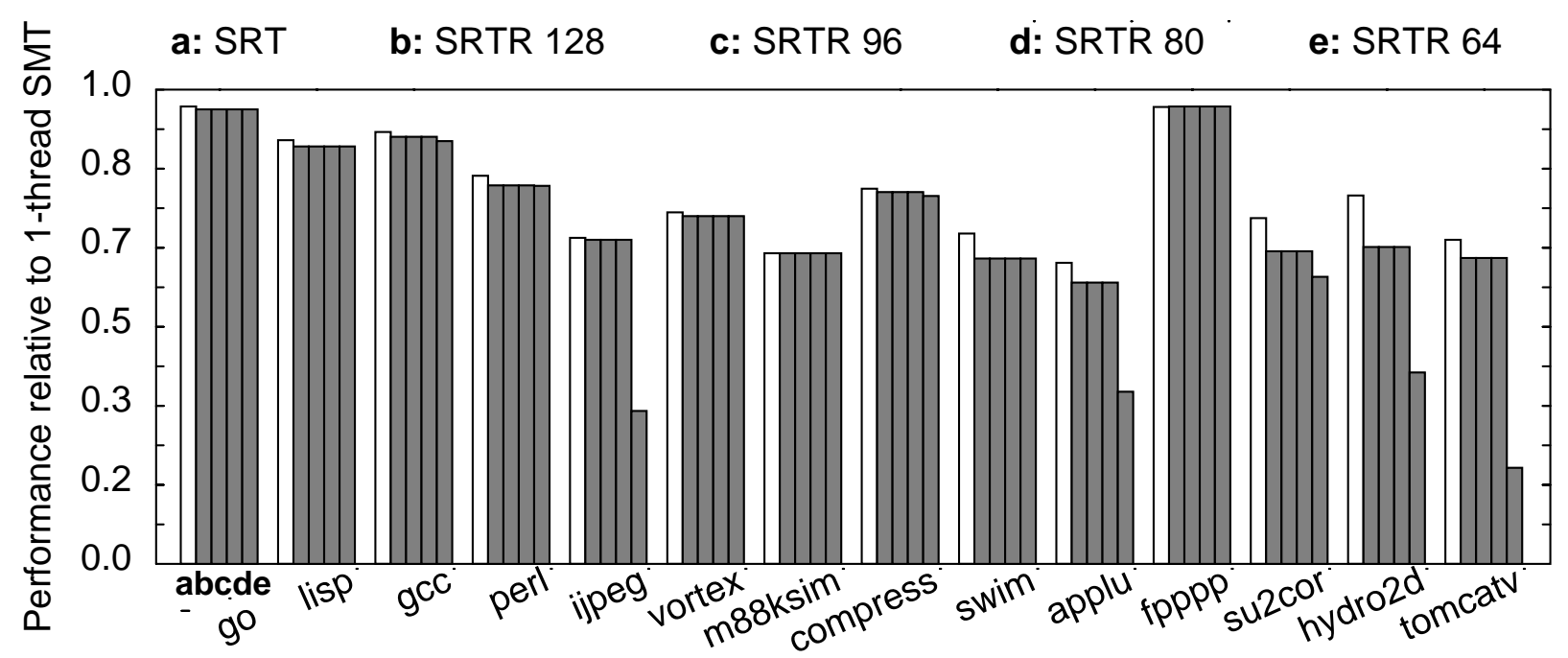

FIGURE 6: Impact of RVQ size.

\begin{tabular}{|l||l|l||l|l||l|}
\hline Benchmark & $\begin{array}{l}\text { Percent } \\
\text { elided }\end{array}$ & Benchmark & $\begin{array}{l}\text { Percent } \\
\text { elided }\end{array}$ & Benchmark & $\begin{array}{l}\text { Percent } \\
\text { elided }\end{array}$ \\
\hline \hline go & 53.1 & vortex & 15.7 & fpppp & 18.4 \\
\hline lisp & 24.7 & m88ksim & 38.4 & su2cor & 40.8 \\
\hline gcc & 41.3 & compress & 38.5 & hydro2d & 39.5 \\
\hline perl & 33.5 & swim & 43.7 & tomcatv & 35.1 \\
\hline ijpeg & 49.4 & applu & 50.1 & AVERAGE & 35.3 \\
\hline
\end{tabular}

Table 4: Percent RVQ accesses elided.

sure the impact of RVQ bandwidth on SRTR without and with DBCE. Loads and stores do not use the RVQ and hence the RVQ bandwidth demand comes solely from the ALU/FPU and branch instructions. Both with and without DBCE, SRTR uses a slack of 32, predQ/LVQ/StB sizes of 48/96/48 entries, and an 80-entry RVQ (which was identified as the best size in the last section). We use a DCQ size of 16 (8 for each thread). We varied the DCQ size and did not find much difference mainly because the chains are broken at branches and branch frequency impacts the chain length more than the DCQ size. Because four RVQ ports are as good as five or more for SRTR without DBCE, we vary the number of RVQ ports as 2, 3, and 4. We use SRT with a slack of 256 as the reference, and show performance normalized to the base SMT executing only the standard program. We show the results in Figure 7.

In Table 4, we show the number of RVQ accesses elided by DBCE as a percentage of all RVQ accesses made without DBCE. On average, DBCE elides $35.3 \%$ of all RVQ accesses in both leading and trailing threads. For most programs, the percentage of elided instructions is high using a DCQ of just 16 entries because the programs have an abundance of register dependences in nearby instructions. The exceptions are vortex and fpppp; both programs have a high fraction (52.8\% and 53.2\%, respectively) of memory instructions. Because loads and stores are not included in the DBCE chains, the programs cannot elide as many instructions as the others. 


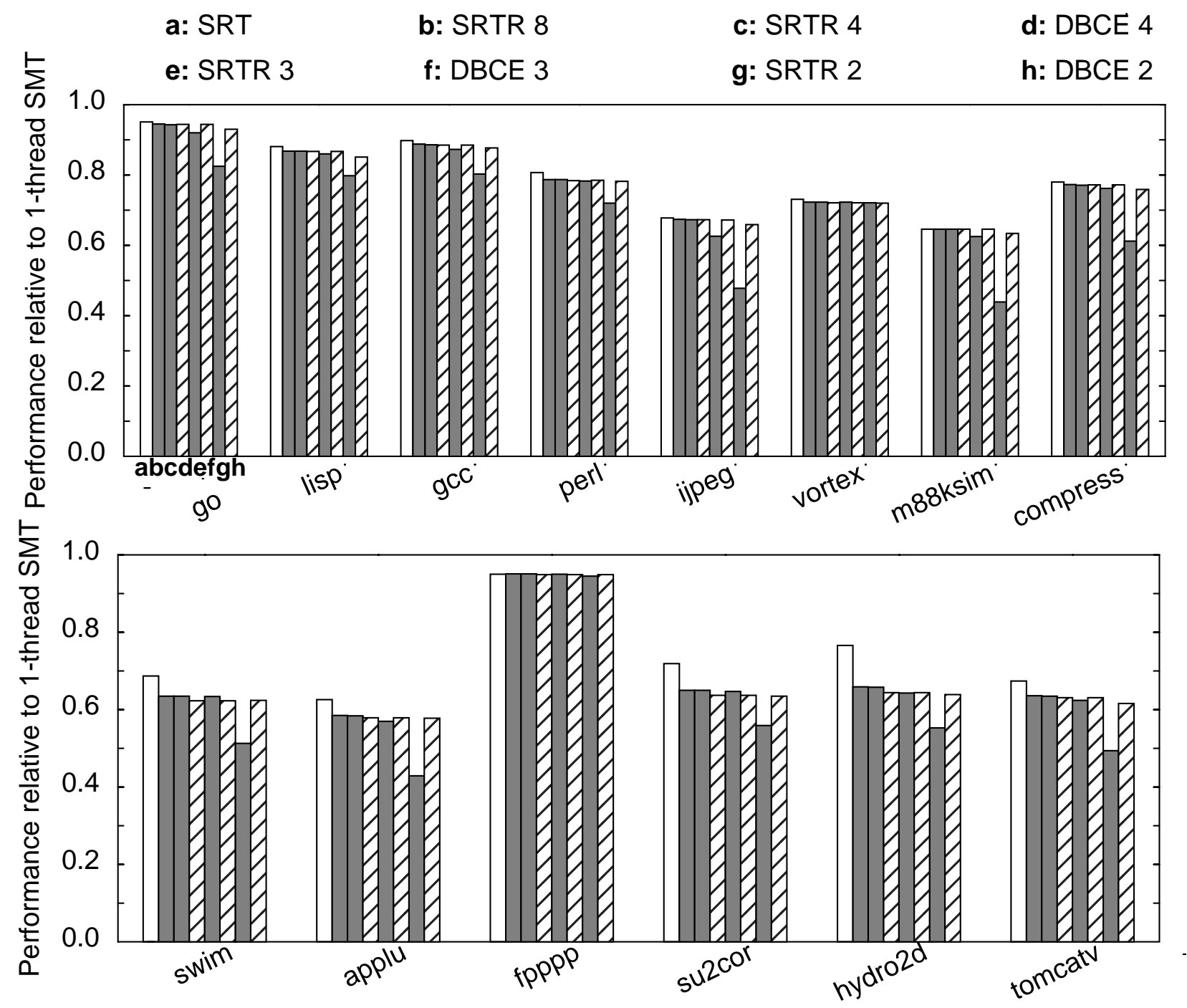

FIGURE 7: Effectiveness of DBCE in reducing RVQ bandwidth demand.

Let us first analyze SRTR performance without DBCE. From Figure 7, we see that for all the programs, a 4ported RVQ (third bar) performs as well as an 8-ported RVQ (second bar). As the number of RVQ ports decreases from 4 to 3 and 2, most programs incur significant performance loss. For the integer programs, performance drops by $2 \%$ and $18 \%$, on average, with 3 and 2 RVQ ports, respectively, compared to 4 RVQ ports. For the FP programs, performance degrades by $1 \%$ and $20 \%$, on average, with 3 and 2 RVQ ports, respectively. vortex and fpppp are the two exceptions that perform as well with 2 RVQ ports as with 4 RVQ, because more than half of the instructions are loads and stores, and do not access the RVQ.

On the other hand, SRTR with DBCE incurs little performance loss even with two RVQ ports. Comparing four ports to two ports, performance degrades by $1 \%$ and $2 \%$ for the integer and FP programs, respectively. A key point to note is that in the case of 4 ports where DBCE is not needed, using DBCE does not degrade performance. This point implies that by exploiting complete to commit time, DBCE avoids stalling the early instructions in the chains waiting for the last instruction in the chain to complete. Looking at SRTR using 2 RVQ 
ports with and without DBCE, DBCE boosts SRTR's performance by 17\% and 18\% for the integer and FP programs, respectively.

\section{Related work}

Watchdog processors are the key concept behind many fault tolerance schemes [5]. The AR-SMT processor is the first to use SMT to execute two copies of the same program [10]. AR-SMT also proposes using speculation techniques to allow communication of data values and branch outcomes between the leading and trailing threads to accelerate execution. A later paper applies the concepts from AR-SMT to CMPs [13]. SRT improves on AR-SMT via the two optimization techniques of slack fetch and checking only stores for an SoR that includes the register file [8]. SRT does not handle recovery. DIVA is another fault-tolerant superscalar processor that uses a simple, in-order checker processor to check the execution of the complex out-of-order processor [1]. DIVA can recover from permanent faults and design errors in the aggressive processor but assumes that no transient faults occur in the checker processor itself. Other work focuses on functional unit fault tolerance [9, $6,4,12]$.

The Compaq NonStop Himalaya [3] and IBM z900 (formerly S/390) [11] employ space-redundancy hardware to achieve fault tolerance. The z900 uses the G5 microprocessor which includes replicated, lock-stepped pipelines. The NonStop Himalaya uses off-the-shelf, lock-stepped microprocessors and compares the external pins on every cycle. In both systems, when the lock-stepped components disagree, the components are stopped to prevent propagation of errors. The z900 uses special microcode to restore program state from a hardware checkpoint module. The NonStop Himalaya does not provide hardware support for recovery. SRT shows that avoiding lock-stepping achieves better performance for fault detection.

\section{Conclusions}

We proposed Simultaneously and Redundantly Threaded processors with Recovery (SRTR) that enhances SRT to include transient fault recovery. SRT's leading instructions may commit before checking for errors, relying on the trailing thread to detect errors. SRTR, on the other hand, must not allow any leading instruction to commit before checking, since a faulty instruction cannot be undone once the instruction commits. To avoid leading instructions stalling at commit for their trailing counterparts, SRTR exploits the time between completion and commit of a leading instruction. SRTR checks as soon as the trailing instruction completes, well before the leading instruction reaches commit. To avoid increasing the bandwidth demand on the register file, SRTR uses the register value queue $(R V Q)$ to hold register values for checking. To reduce the bandwidth pressure on the RVQ itself, SRTR employs dependence-based checking elision (DBCE). By reasoning that faults propagate through dependent instructions, DBCE exploits register (true) dependence chains so that only the last instruction in a chain uses the RVQ to check leading and trailing values. DBCE redundantly builds chains in both the leading and trailing threads and checks its own functionality for faults. 
We evaluated SRTR using the SPEC95 benchmarks. SRTR is within 1\% and 7\% of SRT performance for integer and floating-point programs, respectively. We showed that high prediction accuracies and low off-chip miss rates in the base SMT enable SRTR detection using predictions with a slack of 32 to perform within 5\% of SRT using outcomes with a slack of 256. For our benchmarks, the gap between the average complete to commit time and average memory latency is large enough to allow a slack longer than the average memory latency but shorter than the average complete to commit time. DBCE elides about 35\% of RVQ accesses. SRTR without DBCE incurs about $18 \%$ performance loss on reducing from four (which is equivalent to an unlimited number) to two RVQ ports. With DBCE, a two-ported RVQ performs within $2 \%$ of a four-ported RVQ.

\section{References}

[1] T. M. Austin. DIVA: A reliable substrate for deep-submicron microarchitecture design. In Proceedings of the 32nd Annual International Symposium on Microarchitecture, pages 196-207, Nov. 1999.

[2] D. Burger, T. M. Austin, and S. Bennett. Evaluating future microprocessors: the simplescalar tool set. Technical Report CS TR-1308, University of Wisconsin, Madison, July 1996.

[3] Compaq Computer Corporation. Data integrity for Compaq Non-Stop Himalaya servers. http://nonstop.compaq.com, 1999.

[4] J. G. Holm and P. Banerjee. Low cost concurrent error detection in a VLIW architecture using replicated instructions. In Proceedings of the International Conference on Parallel Processing, 1992.

[5] A. Mahmood and E. J. McCluskey. Concurrent error detection using watchdog processors-A survey. IEEE Trans. on Computers, 37(2):160-174, Feb. 1988.

[6] J. H. Patel and L. Y. Fung. Concurrent error detection on ALU's by recomputing with shifted operands. IEEE Trans. on Computers, 31(7):589-595, July 1982.

[7] J. Ray, J. C. Hoe, and B. Falsafi. Dual use of superscalar datapath for transient-fault detection and recovery. In Proceedings of the 34th annual IEEE/ACM international symposium o n Microarchitecture, Dec. 2001.

[8] S. K. Reinhardt and S. S. Mukherjee. Transient fault detection via simultaneous multithreading. In Proceedings of the 27th Annual International Symposium on Computer Architecture, pages 25-36, June 2000.

[9] D. A. Reynolds and G. Metze. Fault detection capabilities of alternating logic. IEEE Trans. on Computers, 27(12):1093-1098, Dec. 1978.

[10] E. Rotenberg. AR-SMT: A microarchitectural approach to fault tolerance in microprocessors. In Proceedings of Fault-Tolerant Computing Systems, 1999.

[11] T. J. Slegel, et al. IBM's S/390 G5 microprocessor design. IEEE Micro, 19(2):12-23, 1999.

[12] G. S. Sohi, M. Franklin, and K. K. Saluja. A study of time-redundant fault tolerance techniques for high-performance, pipelined computers. In Digest of papers, 19th International Symposium on Fault-Tolerant Computing, pages 436-443, 1989.

[13] K. Sundaramoorthy, Z. Purser, and E. Rotenberg. Slipstream processors: Improving both performance and faulttolerance. In Proceedings of the Ninth International Symposium on Architectural Support for Programming Languages and Operating Systems, pages 257-268. Association for Computing Machinery, Nov. 2000.

[14] D. M. Tullsen, S. J. Eggers, and H. M. Levy. Simultaneous multithreading: maximizing on-chip parallelism. In Proceedings of the 22th Annual International Symposium on Computer Architecture, pages 392-403, June 1995.

[15] S. Wallace, B. Calder, and D. M. Tullsen. Threaded multiple path execution. In Proceedings of the 25th Annual International Symposium on Computer Architecture, pages 238-249, June 1998. 